\title{
Specific fluorogenic substrates for neprilysin (neutral endopeptidase, EC 3.4.24.11) which are highly resistant to serine- and metalloproteases
}

\author{
M.A.S. Medeiros ${ }^{1}$, \\ M.S.F. França ${ }^{1}$, \\ G. Boileau², \\ L. Juliano ${ }^{3}$ and \\ K.M. Carvalho ${ }^{1}$
}

\author{
${ }^{1}$ Laboratório de Neurobiologia Molecular Humana, \\ Departamento de Fisiologia e Farmacologia, Centro de Ciências da Saúde, \\ Universidade Federal do Ceará, Fortaleza, CE, Brasil \\ ${ }^{2}$ Départment de Biochimie, Faculté de Medicine, Université de Montréal, \\ Montréal, Canada \\ ${ }^{3}$ Departamento de Biofísica, Escola Paulista de Medicina, \\ Universidade Federal de São Paulo, São Paulo, SP, Brasil
}

\section{Correspondence \\ K.M. Carvalho \\ Laboratório de Neurobiologia \\ Molecular Humana \\ Departamento de Fisiologia e \\ Farmacologia, CCS, UFCE \\ Caixa Postal 3157 \\ Rua Coronel Nunes de Melo, 1127 \\ 60430-270 Fortaleza, CE \\ Brasil}

Research supported by CNPq, FINEP, FUNCAP, FAPESP, CAPES, and the Medical Research Council of Canada. $\ldots \ldots \ldots \ldots \ldots \ldots \ldots \ldots$

Received April 11, 1997 Accepted August 19, 1997

\begin{abstract}
Two intramolecularly quenched fluorogenic peptides containing $o$ aminobenzoyl (Abz) and ethylenediamine 2,4-dinitrophenyl (EDDnp) groups at amino- and carboxyl-terminal amino acid residues, AbzDArg-Arg-Leu-EDDnp (Abz-DRRL-EDDnp) and Abz-DArg-Arg-PheEDDnp (Abz-DRRF-EDDnp), were selectively hydrolyzed by neutral endopeptidase (NEP, enkephalinase, neprilysin, EC 3.4.24.11) at the Arg-Leu and Arg-Phe bonds, respectively. The kinetic parameters for the NEP-catalyzed hydrolysis of Abz-DRRL-EDDnp and Abz-DRRFEDDnp were $K_{\mathrm{m}}=2.8 \mu \mathrm{M}, k_{\text {cat }}=5.3 \mathrm{~min}^{-1}, k_{\text {cat }} / K_{\mathrm{m}}=2 \mathrm{~min}^{-1} \mu \mathrm{M}^{-1}$ and $K_{\mathrm{m}}=5.0 \mu \mathrm{M}, k_{\text {cat }}=7.0 \mathrm{~min}^{-1}, k_{\mathrm{cat}} / K_{\mathrm{m}}=1.4 \mathrm{~min}^{-1} \mu \mathrm{M}^{-1}$, respectively. The high specificity of these substrates was demonstrated by their resistance to hydrolysis by metalloproteases [thermolysin (EC 3.4.24.2), angiotensin-converting enzyme (ACE; EC 3.4.24.15)], serineproteases [trypsin (EC 3.4.21.4), $\alpha$-chymotrypsin (EC 3.4.21.1)] and proteases present in tissue homogenates from kidney, lung, brain and testis. The blocked amino- and carboxyl-terminal amino acids protected these substrates against the action of aminopeptidases, carboxypeptidases and ACE. Furthermore, $D R$ amino acids ensured total protection of Abz-DRRL-EDDnp and Abz-DRRF-EDDnp against the action of thermolysin and trypsin. Leu-EDDnp and Phe-EDDnp were resistant to hydrolysis by $\alpha$-chymotrypsin. The high specifity of these substrates suggests their use for specific NEP assays in crude enzyme preparations.
\end{abstract}

Neutral endopeptidase (NEP, enkephalinase, neprilysin, EC 3.4.24.11) is a broadly specific zinc metalloendopeptidase which hydrolyzes internal peptide bonds on the amino side of hydrophobic amino acid residues in $\mathrm{P}_{1}{ }_{1}$ position, with Leu or Phe being
Key words

- Neutral endopeptidase

- Enkephalinase

- Neprilysin

- Fluorogenic substrates

- Phosphoramidon the preferred amino acids. Shown to be widely distributed in various tissues, NEP is involved in the regulation and metabolism of a variety of biologically active peptides such as substance $P$, enkephalins, atrial natriuretic factor, bradykinin, gastrin, neurotensin, 
and the chemotactic peptide $(1,2)$.

Several synthetic substrates have been developed to measure NEP activity, such as radiolabeled peptides $\left(\left[{ }^{3} \mathrm{H}\right]\right.$ Leu-enkephalin, D- $\left[{ }^{3} \mathrm{H}\right] \mathrm{Ala}^{2}$-Leu-enkephalin) $(3,4)$, chromogenic peptides (glutaryl-Ala-Ala-Phe2NA, benzyl-Gly-Arg-Arg-Leu-2NA) $(5,6)$ and fluorogenic peptides (dansyl-D-Ala-GlyPhe $\left(\mathrm{pNO}_{2}\right)$-Gly, dansyl-Gly-Phe $\left(\mathrm{pNO}_{2}\right)$ BAla) $(7,8)$.

Although the specificity of all these substrates for NEP is partial, and frequently the use of other protease inhibitors is required during incubation with NEP, they are used to monitor NEP purification, to determine its activity in different tissues under physiological and pathological conditions, as well as to compare the kinetic parameters of different forms of recombinant NEP produced by sitedirected mutagenesis, to develop synthetic inhibitors which have been employed to study the physiological functions of NEP, and for clinical use through the increased level of endogenous peptides that are substrates for the enzyme $(1,2,9)$.

Recently, we described a new intramolecularly quenched fluorogenic substrate for NEP related to Leu-enkephalin, containing $o$-aminobenzoyl (Abz) and ethylenediamine 2,4-dinitrophenyl (EDDnp) groups at aminoand carboxyl-terminal amino acid residues, Abz-GGDFLRRV-EDDnp (10,11). This substrate presents at least one important advantage in relation to other previously described fluorogenic substrates for NEP: its $k_{\text {cat }} / K_{\mathrm{m}}=$ $40 \mathrm{~min}^{-1} \mu \mathrm{M}^{-1}$ is 20 times higher than that of dansyl-D-Ala-Gly-Phe $\left(\mathrm{pNO}_{2}\right)$-Gly (7) and dansyl-Gly-Phe $\left(\mathrm{pNO}_{2}\right)$-BAla (8). Although Abz-GGDFLRRV-EDDnp also presents good specificity for NEP, since it is resistant to other metalloendopeptidases such as angiotensin-converting enzyme (ACE; EC 3.4.24.15) and thermolysin (EC 3.4.24.2), it is partially susceptible to degradation by trypsin-like enzymes which may cleave the $\mathrm{R}-\mathrm{R}$ bond.

In the present study, we document two new intramolecularly quenched fluorogenic substrates for NEP, Abz-DRRL-EDDnp and Abz-DRRF-EDDnp, which were totally resistant to the action of other metalloproteases (ACE, thermolysin), serineproteases (trypsin, EC 3.4.21.4 and chymotrypsin, EC 3.4.21.1) and proteases present in homogenates of several tissues.

The internally quenched fluorogenic peptides Abz-DRRL-EDDnp and Abz-DRRFEDDnp were synthesized by the solution method $(12,13)$. A recombinant soluble form of NEP (rNEP) was expressed using a baculovirus/insect-cell system and purified by immunoaffinity as previously described $(14,15)$.

A crude membrane preparation was obtained from rat tissues as described earlier (10). Briefly, rat tissues were homogenized in 8 volumes (w/v) of $50 \mathrm{mM}$ Tris-HCl buffer, pH 7.5, using a Potter homogenizer, the homogenate was centrifuged for $10 \mathrm{~min}$ at $1,000 \mathrm{~g}$ and the pellet was discarded, the supernatant was centrifuged at $80,000 \mathrm{~g}$ for $60 \mathrm{~min}$, the pellet was washed four times by resuspension in the same buffer used for homogenization and centrifuged under the conditions described above, and the resulting pellet, resuspended in $50 \mathrm{mM}$ Tris- $\mathrm{HCl}$ buffer, pH 7.5 (1/8; w/v), was used as the enzyme source. Protein was measured by the method of Bradford (16). Angiotensin-converting enzyme was purified from guinea pig serum (17).

The synthetic substrates (Abz-DRRLEDDnp and Abz-DRRF-EDDnp) and their enzymatic products were characterized by high-performance liquid chromatography (HPLC) as follows: the substrate $(20 \mathrm{nmol})$ was incubated with purified rNEP (600 ng) or crude enzyme fractions $(1-10 \mu \mathrm{l})$ in a final volume of $100 \mu \mathrm{l} 50 \mathrm{mM}$ Tris- $\mathrm{HCl}$ buffer, $\mathrm{pH} 7.5$, at $37^{\circ} \mathrm{C}$ for $60 \mathrm{~min}$. In inhibition assays, the enzyme was preincubated with 1 $\mu \mathrm{M}$ phosphoramidon for $20 \mathrm{~min}$ before incubation with substrates. The reaction was stopped by heating for $5 \mathrm{~min}$ at $100^{\circ} \mathrm{C}$. After 
centrifugation at $10,000 \mathrm{~g}$ for $10 \mathrm{~min}$, the supernatant fraction was injected into an HPLC column (Nucleosil $5 \mu \mathrm{m} \mathrm{C}_{18}, 145 \mathrm{x}$ $4.5 \mathrm{~mm}$ ) and eluted with a 20-40\% gradient of acetonitrile containing $0.05 \%$ trifluoracetic acid for a period of $50 \mathrm{~min}$, at a flow rate of $1 \mathrm{ml} / \mathrm{min}$. The intact substrate and products, detected by both UV absorbance $(220 \mathrm{~nm})$ and fluorescence $\left(\lambda_{\mathrm{em}}=420 \mathrm{~nm}, \lambda_{\mathrm{ex}}=320\right.$ $\mathrm{nm})$ with the detectors arranged in series, were collected to identify the cleavage site by amino acid analysis (18).

The enzyme assays were monitored by measuring the fluorescence at $\lambda_{\mathrm{em}}=420 \mathrm{~nm}$ and $\lambda_{\mathrm{ex}}=320 \mathrm{~nm}$ with a Shimadzu model F 2000 spectrofluorimeter. The $0.5 \times 1 \mathrm{~cm}$ path-length cuvette containing $500 \mu \mathrm{l}$ of the mixture of $50 \mathrm{mM}$ Tris- $\mathrm{HCl}$ buffer, $\mathrm{pH} 7.5$, and Abz-DRRL-EDDnp $(10 \mu \mathrm{M})$ was placed in the thermostat cell compartment at $37^{\circ} \mathrm{C}$ for 5 min until temperature equilibrium of the solution was attained. When kidney homogenate $(10 \mu \mathrm{l})$ was used, the fluorescence course was recorded continuously for 40 min. For the inhibition assay, the enzyme was preincubated with the inhibitor for 20 $\min$ at $37^{\circ} \mathrm{C}$ before incubation with substrates. The same conditions were used for thermolysin, chymotrypsin, trypsin and ACE at concentrations of $0.15 \mu \mathrm{g} / \mathrm{ml}, 8 \mu \mathrm{g} / \mathrm{ml}, 15$ $\mu \mathrm{g} / \mathrm{ml}$ and $10 \mu \mathrm{g} / \mathrm{ml}$, respectively.

The kinetic parameters for the hydrolysis of Abz-DRRL-EDDnp and Abz-DRRFEDDnp by rNEP were determined from a double-reciprocal Lineweaver-Burk plot. rNEP has an apparent MW of 87,000 (14) and this value was used for the calculation of $k_{\text {cat }}$.

The substrate Abz-DRRL-EDDnp presented the lowest $K_{\mathrm{m}}$ value $(2.8 \mu \mathrm{M})$ similar to $3 \mu \mathrm{M}$ obtained for the longer substrate derived from Leu-enkephalin, Abz-GG DFLRRV-EDDnp $(10,11)$ (Table 1A). Furthermore, although Abz-DRRL-EDDnp presented a lower $k_{\text {cat }} / K_{\mathrm{m}}$ value $\left(2 \mathrm{~min}^{-1} \mu \mathrm{M}^{-1}\right)$ than Abz-GGDFLRRV-EDDnp ( $\left.42 \mathrm{~min}^{-1} \mu \mathrm{M}^{-1}\right)$ $(10,11)$, this value was higher than those of all the short synthetic peptides presented in
Table 1A.

All the substrates presented in Table 1A contain a hydrophobic residue at the $\mathrm{P}_{1}{ }_{1}$ position (Leu, Phe or Val), an essential condition for NEP activity $(1,19)$. Thus, the excellent kinetic parameters of Abz-DRRLEDDnp and Abz-GGDFLRRV-EDDnp may be also explained by interactions of their groups with subsites on the surface of NEP. In these substrates, the presence of EDDnp, a hydrophobic group at position $\mathrm{P}_{2}$, may suggest a strong interaction with an $\mathrm{S}_{2}{ }_{2}$ subsite of NEP.

Table 1B shows the activity of NEP, other metalloendopeptidases (ACE and thermolysin) and serineproteases (trypsin and $\alpha$ chymotrypsin) toward Abz-DRRL-EDDnp, Abz-DRRF-EDDnp and several other synthetic peptides. In contrast to all the other substrates, only Abz-DRRL-EDDnp and AbzDRRF-EDDnp were totally resistant to the action of ACE, thermolysin, trypsin and $\alpha$ chymotrypsin. Furthermore, the activity toward Abz-DRRL-EDDnp and Abz-DRRFEDDnp present in kidney homogenate (Figure 1) and in tissue homogenates of lung, brain and testis (data not shown) was totally inhibited by $1 \mu \mathrm{M}$ phosphoramidon (a highly specific NEP inhibitor), showing also the high resistance of these substrates to other tissue proteases.

It has been previously demonstrated that the Abz and EDDnp groups at amino- and carboxy-terminal amino acid residues protect peptides against the action of aminopeptidases, carboxypeptidases and ACE $(10,11)$. However, these groups do not protect peptides against the action of thermolysin or trypsin, since the substrate Abz-RRLEDDnp, containing $\mathrm{R}$ at position $\mathrm{P}_{2}$, was hydrolyzed by these enzymes (data not shown). Thus, the presence of $\mathrm{DR}$ at position $\mathrm{P}_{2}{ }_{2}$ ensured a total protection of Abz-DRRLEDDnp and Abz-DRRF-EDDnp against thermolysin and trypsin. Finally, it may be suggested that EDDnp ensures a total protection of the L-EDDnp and F-EDDnp bonds against 
Table 1 - A, Comparison of the kinetic parameters for hydrolysis of Abz-DRRL-EDDnp and Abz-DRRF-EDDnp by NEP with those of Leu-enkephalin and other synthetic substrates used in current NEP assays. ${ }^{a}$ Mean values \pm SEM of four independent determinations. The arrows indicate the peptide bonds cleaved by NEP.

B, Incubation of Abz-DRRL-EDDnp, Abz-DRRF-EDDnp, Abz-GGdFLRRV-EDDnp and other synthetic substrates with NEP $(0.5 \mu \mathrm{g} / \mathrm{ml})$, ACE $(10 \mu \mathrm{g} / \mathrm{ml})$, thermolysin $(0.15 \mu \mathrm{g} / \mathrm{ml})$, trypsin $(15 \mu \mathrm{g} / \mathrm{ml})$, and chymotrypsin $(8 \mu \mathrm{g} / \mathrm{ml})$. Reactions were carried out at $\mathrm{pH} 7.5$ and $37^{\circ} \mathrm{C}$. Concentration was $10 \mu \mathrm{M}$ for substrates 1,2 and 3 . nd, Not determined.

\begin{tabular}{|c|c|c|c|c|c|c|}
\hline \multicolumn{7}{|l|}{$A$} \\
\hline Substrates & \multicolumn{2}{|l|}{$K_{\mathrm{m}}(\mu \mathrm{M})$} & $k_{\text {cat }}\left(\mathrm{min}^{-1}\right)$ & \multicolumn{2}{|c|}{$k_{\text {cat }} / K_{\mathrm{m}}\left(\mathrm{min}^{-1} \mu \mathrm{M}^{-1}\right)$} & References \\
\hline (1) Abz-DR-R $\downarrow$ L-EDDnp & $2.8 \pm 1.5^{a}$ & & 5.3 & & & - \\
\hline (2) Abz-DR-R $\downarrow$ F-EDDnp & $5 \pm 1.5^{\mathrm{a}}$ & & 7 & & & - \\
\hline (3) Abz-G-G-F-L-R-R $\downarrow$ V-EDDnp & $17 \pm 1.5^{\mathrm{a}}$ & & 736 & & & 10,11 \\
\hline (4) Abz-G-G-DF-L-R-R $\downarrow$ V-EDDnp & $3 \pm 2^{a}$ & & 127 & & & 10,11 \\
\hline \multirow[t]{3}{*}{ (5) Y-DA-G $\downarrow$ F-L } & 22 & & 508 & & & 14 \\
\hline & 19 & & - & & & 20 \\
\hline & 49 & & - & & & 4 \\
\hline (6) Glutaryl-A-A $\downarrow$ F-2NA & 590 & & 592 & & & 5,6 \\
\hline (7) Benzyl-G-R-R $\downarrow$ L-2NA & 180 & & 240 & & & 5 \\
\hline (8) Dansyl-DA-G $\downarrow$ F-(pNO 2$)-G$ & 45 & & 59 & & & 7 \\
\hline (9) Dansyl-G $\downarrow$ F-(pNO 2$)-3 A$ & 37 & & 68 & & & 8 \\
\hline (10) Suc-A-A $\downarrow$ F-NH-Np & 76 & & 2.19 & & & 19 \\
\hline (11) Suc-A-A $\downarrow$ L-NH-Np & 5.1 & & 290 & & & 19 \\
\hline (12) Suc-P-A $\downarrow$ F-NH-Np & 0.905 & & 50 & & & 19 \\
\hline \multicolumn{7}{|l|}{ B } \\
\hline \multicolumn{7}{|c|}{ endopeptidase } \\
\hline (1) Abz-DR-R-L-EDDnp & + & - & - & - & - & - \\
\hline (2) Abz-DR-R-F-EDDnp & + & - & - & - & - & - \\
\hline (3) Abz-G-G-DF-L-R-R-V-EDDnp & + & - & - & + & - & 10,11 \\
\hline (4) Suc-A-A-F-NH-Np & + & nd & + & nd & + & 19 \\
\hline (5) Suc-A-A-L-NH-Np & + & nd & + & nd & + & 19 \\
\hline (6) Suc-P-A-F-NH-Np & + & nd & + & nd & + & 19 \\
\hline
\end{tabular}

the action of $\alpha$-chymotrypsin, since no amino acid is involved.

We have previously shown that the intramolecularly quenched fluorogenic peptide Abz-GGDFLRRV-EDDnp can be used for a rapid, highly selective and sensitive NEP assay $(10,11)$. Indeed, since the catalytic constants obtained with Abz-GG DFLRRV-EDDnp are better than those obtained with Abz-DRRL-EDDnp, Abz-DRRFEDDnp and other synthetic peptides shown here, it is the best substrate for use in NEP assays. As shown in Table 1B, although resistant to hydrolysis by ACE, thermolysin and $\alpha$-chymotrypsin, Abz-GGDFLRRVEDDnp was hydrolyzed at the R-R bond by trypsin, suggesting that it may also be susceptible to hydrolysis by trypsin-like enzymes present in some crude enzyme preparations. Taken together, these results show that Abz-DRRL-EDDnp and Abz-DRRFEDDnp are substrates more specific for NEP than Abz-GGDFLRRV-EDDnp.

Finally, the results presented in this study 


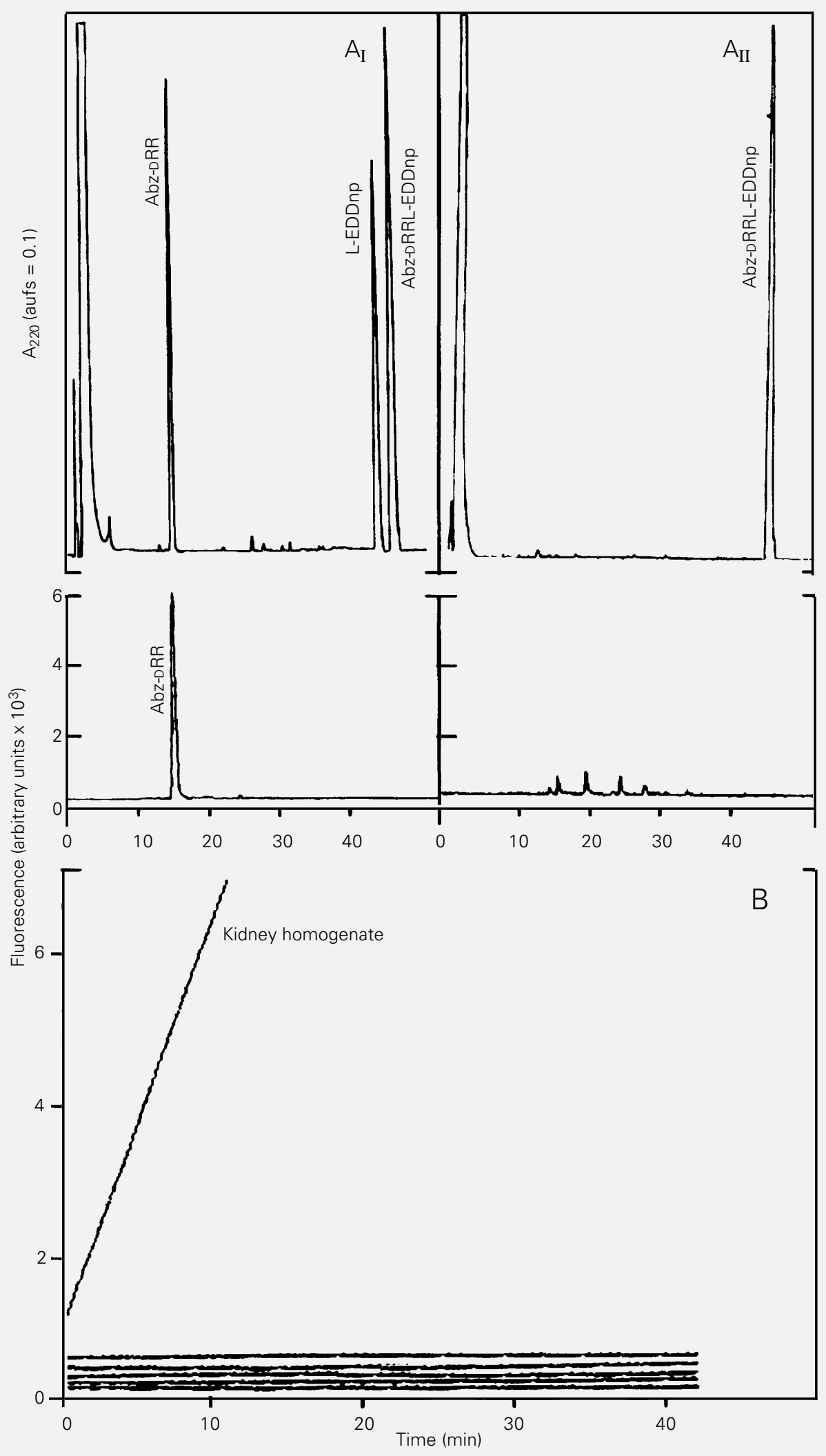

Figure 1 - A, HPLC elution profile of Abz-DRRL-EDDnp after hydrolysis by rat kidney homogenate $\left(A_{1}\right)$ and inhibition of the reaction by phosphoramidon $\left(A_{\| I}\right)$. The substrate $(20 \mathrm{nmol})$ was incubated with $10 \mu$ l kidney homogenate $(180 \mu \mathrm{g} / \mathrm{ml})$ for 60 min in $100 \mu \mathrm{l} 50 \mathrm{mM}$ Tris-HCl buffer, $\mathrm{pH} 7.5$, at $37^{\circ} \mathrm{C}$, in the presence or absence of $1 \mu \mathrm{M}$ phosphoramidon. The reaction was stopped by heating the mixture at $100^{\circ} \mathrm{C}$ for $5 \mathrm{~min}$ followed by centrifugation at $10,000 \mathrm{~g}$ for $10 \mathrm{~min}$. An aliquot of the supernatant (100 $\mu$ l) corresponding to $90 \%$ of the reaction mixture was submitted to HPLC as described in the text. $B$, Continuous fluorescence recording $\left(\lambda_{\mathrm{em}}=420\right.$ $\left.\mathrm{nm}, \lambda_{\mathrm{ex}}=320 \mathrm{~nm}\right)$ of the hydrolysis of Abz-DRRL-EDDnp (10 $\mu \mathrm{M})$ by the enzymes presented from top to bottom: kidney homogenate $(10 \mu \mathrm{l})$ in the absence or presence of $1 \mu \mathrm{M}$ phosphoramidon, ACE $(10 \mu \mathrm{g} / \mathrm{ml})$, thermolysin $(0.15 \mu \mathrm{g} / \mathrm{ml})$, trypsin (15 $\mu \mathrm{g} / \mathrm{ml}$ ) and $\alpha$-chymotrypsin (8 $\mu \mathrm{g} / \mathrm{ml})$. All incubations were performed in a final volume of 500 $\mu 50 \mathrm{mM}$ Tris- $\mathrm{HCl}$ buffer, $\mathrm{pH}$ 7.5 , at $37^{\circ} \mathrm{C}$. 
lead us to the following conclusions: i) the previously described substrate AbzGGDFLRRV-EDDnp $(10,11)$, which presents the best kinetic parameters, is more suitable than Abz-DRRL-EDDnp and Abz-DRRFEDDnp for NEP assays in purified enzyme preparations; ii) the new substrates AbzDRRL-EDDnp and Abz-DRRF-EDDnp, which present the best specificities, are more suitable than Abz-GGDFLRRV-EDDnp for NEP assays in crude enzyme preparations.

\section{References}

1. Roques BP, Noble F, Daugé $V$, FourniéZaluski MC \& Beaumont A (1993). Neutral endopeptidase 24.11: structure, inhibition, and experimental and clinical pharmacology. Pharmacological Reviews, 45: 87-146.

2. Erdös EG \& Skidgel RA (1989). Neutral endopeptidase 24.11 (enkephalinase) and related regulators of peptide hormones. FASEB Journal, 3: 145-151.

3. Vogel Z \& Altstein M (1977). The adsorption of enkephalin to porous polystyrene beads: a simple assay for enkephalin hydrolysis. FEBS Letters, 80: 332-335.

4. Llorens C, Malfroy B, Schwartz JC, Gacel G, Roques BP, Roy J, Morgat JL, JavoyAgid F \& Agid $Y$ (1982). Enkephalin dipeptidyl carboxypeptidase (enkephalinase) activity: selective radioassay, properties, and regional distribution in human brain. Journal of Neurochemistry, 39: 10811089.

5. Almenoff J, Wilk S \& Orlowisky M (1981). Membrane bound pituitary metalloendopeptidase: apparent identity to enkephalinase. Biochemical and Biophysical Research Communications, 102: 206-214.

6. Almenoff J, Wilk S \& Orlowisky M (1984). Biochemical and immunological properties of a membrane-bound brain metalloendopeptidase: comparison with thermolysin-like kidney neutral metalloendopeptidase. Journal of Neurochemistry, 42: 151-157.

7. Florentin D, Sassi A \& Roques BP (1984). A highly sensitive fluorogenic assay for "enkephalinase", a neutral metalloendopeptidase that releases tyrosin-glycineglycine from enkephalins. Analytical Biochemistry, 141: 62-69.
8. Goudreau N, Guis C, Soleilhac JM \& Roques BP (1994). Dns-Gly-(p-NO $\left.\mathrm{N}_{2}\right)$ PheB-Ala, a specific fluorogenic substrate for neutral endopeptidase 24.11. Analytical Biochemistry, 219: 87-95.

9. Gros C, Souque A, Schwartz JC, Duchier J, Cournot A, Baumer P \& Lecomet JM (1989). Protection of atrial natriuretic factor against degradation, diuretic and natriuretic responses after in vivo inhibition of enkephalinase (EC 3.4.24.11) by acetorphan. Proceedings of the National Academy of Sciences, USA, 86: 7580-7584.

10. Carvalho KM, Boileau G, França MSF, Medeiros MAS, Camargo ACM \& Juliano $L$ (1995). A new fluorimetric assay for neutral endopeptidase (EC 3.4.24.11). Brazilian Journal of Medical and Biological Research, 28: 1055-1059.

11. Carvalho KM, Boileau G, Camargo ACM \& Juliano $L$ (1996). A highly selective assay for neutral endopeptidase based on the cleavage of a fluorogenic substrate related to leu-enkephalin. Analytical Biochemistry, 237: 167-173.

12. Chagas JR, Juliano L \& Prado ES (1990). Intramolecularly quenched fluorogenic tetrapeptide substrates for tissue and plasma kallikreins. Analytical Biochemistry, 192: 419-425.

13. Oliveira MCF, Hirata IY, Chagas JR, Boschov P, Gomes RAS, Figueredo AFS \& Juliano L (1992). Intramolecularly quenched fluorogenic peptide substrates for human renin. Analytical Biochemistry, 203: 39-46.

14. Fossiez F, Lemay G, Labonté N, Parmentier-Lesage $F$, Boileau $G$ \& Crine $P$ (1992). Secretion of a functional form of neutral endopeptidase-24.11 from a baculovirus-infected insect cell line. Biochemical Journal, 284: 53-59.
15. Lemay G, Waksman G, Roques BP, Crine P \& Boileau G (1989). Fusion of a cleavable signal peptide to the ectodomain of neutral endopeptidase (EC 3.4.24.11) results in the secretion of an active enzyme in COS-1 cells. Journal of Biological Chemistry, 264: 15620-15623.

16. Bradford MM (1976). A rapid and sensitive method for the quantitation of microgram quantities of protein utilizing the principle of protein-dye binding. Analytical Biochemistry, 72: 248-254.

17. Dorer FE, Skeggs LT, Kahn JR, Lentz KE \& Levine M (1970). Angiotensin converting enzyme: method of assay and partial purification. Analytical Biochemistry, 33: 102-103.

18. Carvalho KM, Joudiou C, Bousseta $H_{\text {, }}$ Leseney AM \& Cohen $P$ (1992). A peptide-hormone-inactivating endopeptidase in Xenopus laevis skin secretion. Proceedings of the National Academy of Sciences, USA, 89: 84-88.

19. Spungin-Bialik A, Ben-Meir D, Fudim E, Carmeli S \& Blumberg S (1996). Sensitive substrates for neprilysin (neutral endopeptidase) and thermolysin that are highly resistant to serine proteases. FEBS Letters, 380: 79-82

20. Malfroy B \& Schwartz JC (1982). Properties of "enkephalinase" from rat kidney: comparison of dipeptidyl-carboxypeptidase and endopeptidase activities. Biochemical and Biophysical Research Communications, 106: 276-285. 